\title{
Rapid and Sensitive Detection of Lung Cancer Biomarker Using Nanoporous Biosensor Based on Localized Surface Plasmon Resonance Coupled with Interferometry
}

\author{
Jae-Sung Lee, ${ }^{1}$ Sae-Wan Kim, ${ }^{1}$ Eun-Yoon Jang, ${ }^{2}$ Byoung-Ho Kang, ${ }^{3}$ Sang-Won Lee, \\ Gopalan Sai-Anand, ${ }^{1}$ Seung-Ha Lee, ${ }^{4}$ Dae-Hyuk Kwon, ${ }^{5}$ and Shin-Won Kang ${ }^{1}$ \\ ${ }^{1}$ School of Electronics Engineering, College of IT Engineering, Kyungpook National University, Daegu 702-701, Republic of Korea \\ ${ }^{2}$ Gyeongbuk Institute of IT Convergence Industry Technology, Gyeongsangbuk-do 712-832, Republic of Korea \\ ${ }^{3}$ Center for Functional Devices Fusion Platform, Kyungpook National University, Daegu 702-701, Republic of Korea \\ ${ }^{4}$ Department of Biomedical, School of Medicine, Dankook University, Chungnam 330-714, Republic of Korea \\ ${ }^{5}$ Department of Electronics Engineering, Kyungil University, Gyeongsangbuk-do 712-702, Republic of Korea
}

Correspondence should be addressed to Shin-Won Kang; swkang@knu.ac.kr

Received 22 May 2015; Accepted 15 July 2015

Academic Editor: Anh-Tuan Le

Copyright (C) 2015 Jae-Sung Lee et al. This is an open access article distributed under the Creative Commons Attribution License, which permits unrestricted use, distribution, and reproduction in any medium, provided the original work is properly cited.

\begin{abstract}
We propose a nanobiosensor to evaluate a lung cancer-specific biomarker. The nanobiosensor is based on an anodic aluminum oxide (AAO) chip and functions on the principles of localized surface plasmon resonance (LSPR) and interferometry. The poredepth of the fabricated nanoporous AAO chip was $1 \mu \mathrm{m}$ and was obtained using a two-step electrochemical anodization process. The sensor chip is sensitive to the refractive index (RI) changes of the surrounding medium and also provides simple and labelfree detection when specific antibodies are immobilized on the gold-deposited surface of the AAO chip. In order to confirm the effectiveness of the sensor, the antibodies were immobilized on the surface of the AAO chip, and the lung cancer-specific biomarker was applied atop of the immobilized-antibody layer using the self-assembled monolayer method. The nanoporous AAO chip was used as a sensor system to detect serum amyloid Al, which is a lung cancer-specific biomarker. The specific reaction of the antigenantibody contributes to the change in the RI. This in turn causes a shift in the resonance spectrum in the refractive interference pattern. The limit of detection (LOD) was found to be $100 \mathrm{ag} / \mathrm{mL}$ and the biosensor had high sensitivity over a wide concentration range.
\end{abstract}

\section{Introduction}

Highly sensitive and rapid detection of biomarkers is essential for biosensors used in medical diagnostics, genetics, or environmental monitoring applications $[1,2]$. Nanotechnology based biosensor devices have the potential to overcome many of the disadvantages of conventional health diagnostic and monitoring methods. Among various nanotechnology based biosensors, electrochemical nanoscale biosensors offer the ability to measure biomedical parameters directly and rapidly, without using fluorescent labels. Nanoscale sensors also offer the potential for in vivo sensing.

The development of ultrasensitive sensing and biosensing devices using nanomaterials and nanostructures received a great deal of attention in the last decade, primarily because of their unique physical and chemical properties. Nanobiosensors, in particular, are analytical devices that utilize nanoscale detector components to identify minute biological elements with enhanced sensitivity [3]. These biosensors are important in the field of biotechnology because of their direct, faster, more accurate, and more selective detection of the analyte at low concentrations $[4,5]$.

Recently, considerable research has been carried out to develop nanobiosensors using nanostructures, such as nanofibers, nanoparticles, nanowires, nanorods, and nanotubes. These nanostructures are important in the manufacturing of the biosensor because of their unique electronic, optical, magnetic, thermoelectrical, and chemical properties [6-9]. 
The nanostructures in these biosensors are highly selective and sensitive; hence, they are used for medical diagnosis, monitoring of diseases, drug discovery, and the detection of environmentally relevant biological agents [10].

The combination of nanostructures and biomolecules is of considerable interest in the field of nanobiotechnology. Fundamentally, biosensors are based on the coupling of a ligand-receptor binding reaction to a signal transducer [11]. Several techniques for the detection of biomolecules based on the ligand-receptor binding reaction have been reported. These include techniques, such as optical sensors using light $[12,13]$, semiconductor sensors using changes in electric properties due to an external stimulus, and chemical sensors using chemical changes $[14,15]$. Although each of these techniques has its individual strengths and weaknesses, a strong case has been made that the optical type biosensors have greater potential. In particular, techniques that are based on localized surface plasmon resonance (LSPR) on golddeposited nanostructures are fast becoming the methods of choice in many affinity biosensing applications [16, 17]. LSPR has been used to monitor a broad range of analytesurface binding interactions, including the adsorption of small molecules, ligand-receptor binding, protein adsorption on self-assembled monolayers (SAMs), antibody-antigen binding, and protein-DNA interactions $[18,19]$.

All of these properties make anodic aluminum oxide (AAO) an excellent sensing platform with exciting opportunities in the development of advanced, simple, smart, and cost-effective sensing devices that may be used in numerous analytical applications. In addition, AAO chip has a unique set of chemical, optical, mechanical and electrical properties. These properties include chemical resistance, thermal stability, biocompatibility, and a large surface area. The AAO chips can be synthesized via anodization of metal aluminum. Using this procedure, precise control of the pore diameter and length can be achieved [20]. Owing to these fairly welldefined nanopores, AAO chips have been used in popular applications in many areas, such as nanostructured-material preparation and biological and chemical separations [21, 22]. The use of AAO in these for applications has a lot of potential in future developments and in the translation of these research products into commercial devices.

In our previous work, we confirmed that AAO chips can be used to detect biomolecules [23, 24]. Furthermore, we successfully fabricated a biosensor where the controlled poredepth and a uniform surface could be retained. The AAO chip responses were evaluated by the surface RI changes through the interaction between the interference and LSPR.

In this study, we describe major advances and developments in the lung cancer-specific biomarker sensor system based on a nanoporous AAO chip. The lung cancer-specific biomarker of choice was serum amyloid A1 (SAA1). Herein, we will briefly describe the nanoporous structures of AAO prepared at $1 \mu \mathrm{m}$ of pore-depth, various pore diameters, ranging from $15 \mathrm{~nm}$ to $95 \mathrm{~nm}$, using a two-step electrochemical anodization procedure. We also describe their key optical and electrochemical properties, which make AAO ideal for specific sensing applications. The pore diameters of the AAO chip were tuned using a pore-widening treatment as a function of the anodization conditions. The sensor chip is sensitive to the refractive index (RI) of the surrounding medium and also provides simple and label-free detection when specific antibodies are immobilized to the gold-deposited surface of AAO chip. The specific antigen-antibody reaction contributes to the change in the RI that causes a shift in the resonance spectrum of the refractive interference pattern. The results indicate that the pore size has to be large enough to allow biomolecules to enter the pores freely, but small enough to retain the optical reflectivity of the AAO membrane. The limit of detection (LOD) of our sensor system for SAA1 was at a concentration of $100 \mathrm{ag} / \mathrm{mL}$. Our proposed AAO chip has high sensitivity and real-time detection of biomolecular interactions. These properties allow these biosensors to be applied in a wide array of applications.

\section{Experimental Section}

2.1. Materials. Aluminum sheet, perchloric acid, ethanol, oxalic acid, phosphoric acid, chromic acid, 11-mercapto-1undecanol acid, N-(3-dimethylaminopropyl)- $\mathrm{N}^{\prime}$-ethylcarbodiimide hydrochloride (EDC), N-hydroxysuccinimide (NHS), and dimethyl sulfoxide (DMSO) were supplied by Sigma-Aldrich (USA). Buffer solution of Tris-HCl ( $\mathrm{pH} 7.4$ ) (WELGENE Inc. Korea) was used.

2.2. Serum Amyloid A (SAA). SAA is a high density lipoprotein- (HDL-) associated apolipoprotein and is known to play a major role in the modulation of inflammation and in the metabolism and transport of cholesterol [25]. It has been recently proposed that SAA is a potentially useful biomarker to monitor patients harboring human tumors, including gastric and nasopharyngeal cancer [26, 27]. Moreover, in lung cancer patients, using mass spectrometry and proteomic technologies, the SAA was identified as the top differentially expressed protein that was able to differentiate the serum of patients from the serum of healthy individuals. Among four human SAA gene families, the acute SAAs, SAA1 and SAA2, are known to be induced up to several hundredfold. SAA3 is a pseudogene whereas the SAA4 is constantly expressed $[28,29]$. In the present report, we propose that SAA1 is a good serum biomarker for the detection of lung cancer.

2.3. Principle of Sensor Operation and Structure. In order to increase the sensitivity of the sensor system, we developed a sensor chip designed to detect the SAA1 antigen (ProtanBio, Korea) using both interferometry and LSPR. Gold was deposited on a nanoporous structure to induce LSPR and to allow immobilization of a SAA1 antibody $(100 \mu \mathrm{g} / \mathrm{mL}$, ProtanBio, Korea). When the light is vertically incident, the interference fringe pattern appears as the difference in optical path between the upper part of the gold-deposited nanostructure and the lower part of the core. The reaction of the SAA1 antigen with the SAA1 antibody using the SAM method causes a change in the wavelength-dependent refractive index of the sensing membrane. The wavelength shift and the change in reflectance intensity are caused by 
TABLE 1: Parameters for fabrication of various pore diameters of AAO chip.

\begin{tabular}{lccc}
\hline Sample & Electrolyte & Anodization voltage $(\mathrm{V})$ & 2nd anodization time \\
\hline (a) & 0.3 M oxalic acid & 20 & 50 \\
(b) & 0.3 M oxalic acid & 40 & 16 \\
(c) & 0.4 M oxalic acid & 50 & 7 \\
(d) & 0.1 M phosphoric acid & 50 & 190 \\
(e) & 1 M phosphoric acid & 50 & 60 \\
\hline
\end{tabular}

the interference. Thus, the target material is measured by the effective optical thickness $(L)$ of the detection membrane and spectrum change in reflection light from RI.

Effective $L$ was demonstrated by [30] to be the multiple of $\mathrm{RI}$ and thickness by the following equation:

$$
\begin{aligned}
m \lambda_{1} & =2 n_{\lambda_{1}} L, \\
(m+1) \lambda_{2} & =2 n_{\lambda_{2}} L .
\end{aligned}
$$

Here, $m$ is the order of interference meaning optical path length difference and phase shift of reflection light; $\lambda$ is the wavelength of light; $n_{\lambda}$ is the effective RI at the wavelength $\lambda$ [30]. $\lambda_{1}$ and $\lambda_{2}$ show interference neighboring waves of orders $m$ and $m+1$, respectively. Because the band gap of the AAO membrane is large $n_{\lambda}$ it can be expressed as a constant at long wavelength bandwidth. Thus, the relationship between fringe order $m, \lambda_{1}$, and $\lambda_{2}$ can be given by the following equation:

$$
\frac{\lambda_{1}}{\lambda_{2}}=\frac{(m+1)}{m} .
$$

To enhance the sensors sensitivity, several research groups focused on nanoscale optical sensors using nanostructures and nanoparticles. Thus, the proposed LSPR-based biosensor depends on the shape and size of gold nanostructure deposited on the AAO membrane. The LSPR phenomenon can be understood using the RI response model of propagation surface plasmon on a metal surface [31]:

$$
\Delta \lambda_{\max }=m \Delta n\left[1-e^{\left(-2 d / l_{d}\right)}\right] .
$$

Here, $\Delta \lambda_{\max }$ is the wavelength shift, $m$ is the refractive sensitivity, $\Delta n$ is the change in the RI by optical adsorption, $d$ is the effective thickness of the adsorption surface, and $l_{d}$ is the characteristic electromagnetic field decay length. As shown in (1), the RI and $L$ of detection membrane are changed when the analyte on the surface of metal structure is detected by the antigen-antibody reaction. In addition, based on (3), extremely small changes such as the change in the RI of the surrounding media can be detected using LSPR by the antigen-antibody reaction. In the present report, the proposed sensing system causes a significant change in sensitivity, as observed by both optical LSPR and interferometry. A schematic diagram of the fabricated AAO chip and the optical detection method is shown in Figure 1.

2.4. Fabrication of AAO Chip. AAO chip can be obtained using a two-step anodization process of the aluminum sheet

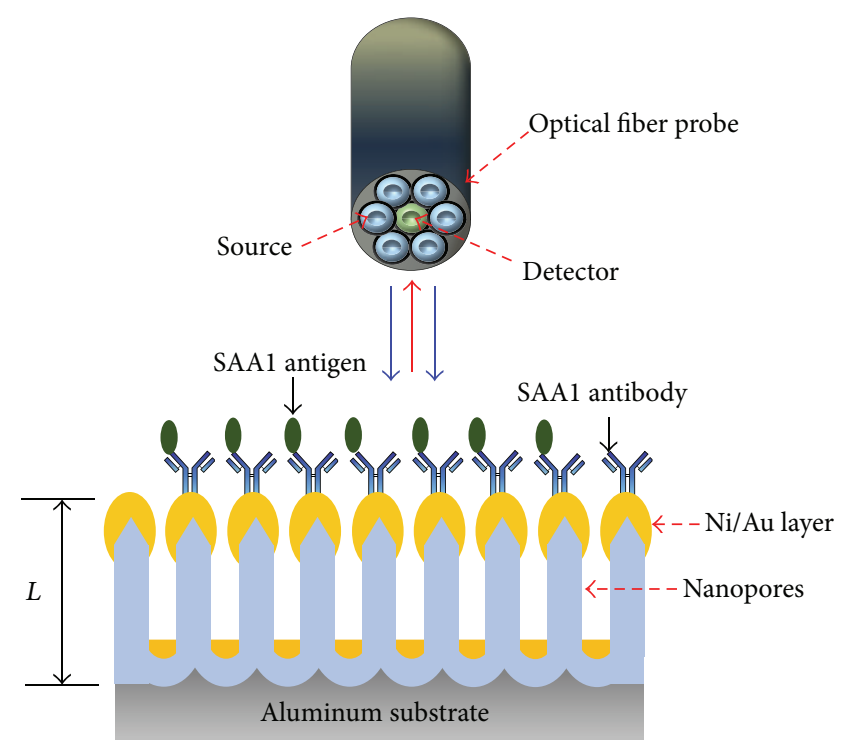

FIGURE 1: Schematic diagram of the fabricated AAO chip and the optical detection method.

that was anodized using electrolytes [32]. In order to obtain various diameters of the pore, the AAO chip was controlled by changing the anodization conditions, such as the applied voltage, the anodization time, and the types of electrolyte acids. The composition of the parameters for fabrication of nanoporous AAO chip is given in Table 1. Initially, the aluminum sheet was placed in a mixed solution of $70 \%$ perchloric acid and $30 \%$ ethanol and a voltage of $20 \mathrm{~V}$ was applied to remove impurities, the native oxide layer, and to smoothen the surface of the aluminum sheet. Second, to generate the oxide layer, the aluminum sheet was cleaned and was placed in a different electrolyte acid (0.3, 0.4 M oxalic acid and 0.1, $1 \mathrm{M}$ phosphoric acid) and applied with a bias for each different voltage setting for $30 \mathrm{~min}$. The anodization process was maintained at $9^{\circ} \mathrm{C}$ in a circulator system. Third, to remove the first AAO layer, the aluminum sheet was placed in a solution that consisted of $6 \mathrm{wt} \%$ phosphoric acid and $1.8 \mathrm{wt} \%$ chromic acid for $90 \mathrm{~min}$ at $60^{\circ} \mathrm{C}$. Finally, the second anodization process was performed using the same conditions as in the first anodization.

The AAO chip was fabricated with different diameters of the pore by controlling the anodization conditions. We measured the pore diameter and surface uniformity using 


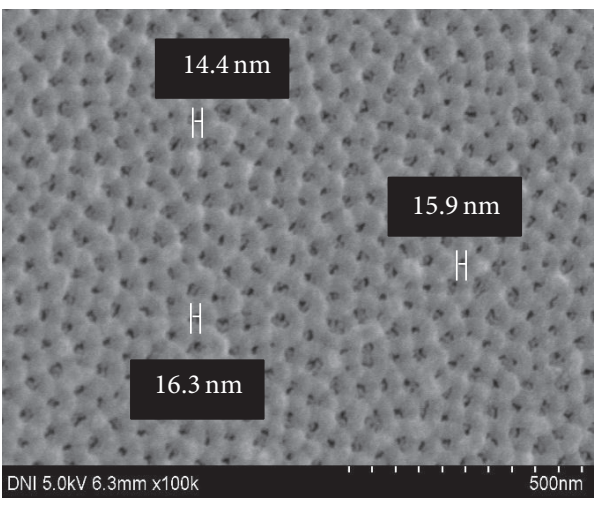

(a)

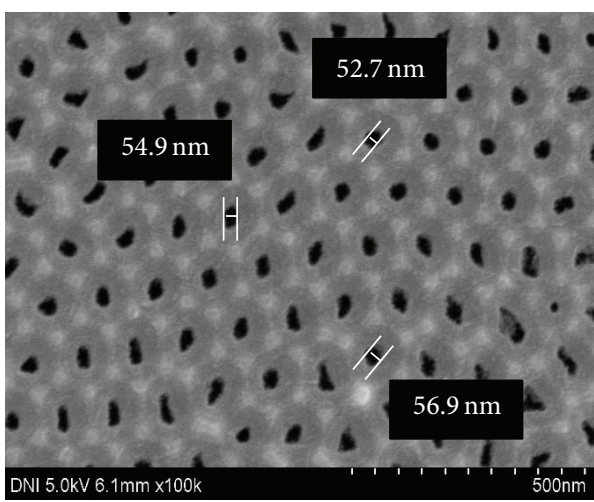

(c)

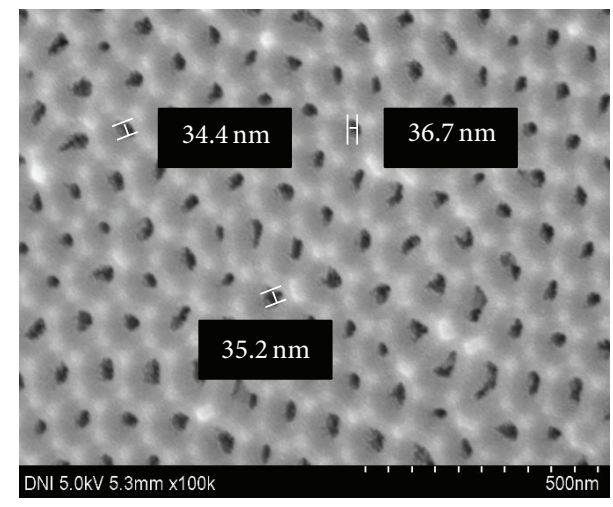

(b)

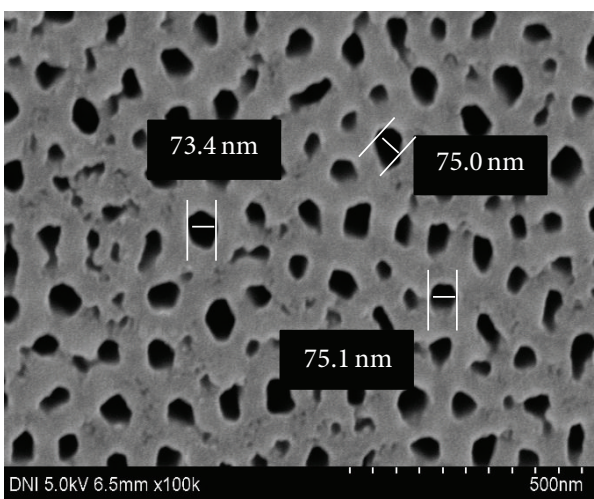

(d)

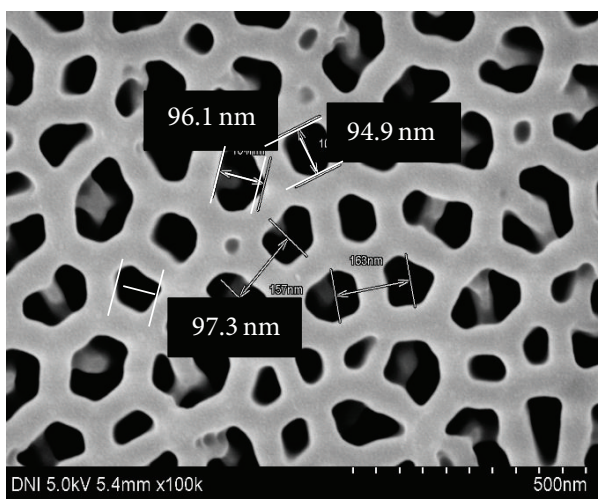

(e)

FIGURE 2: SEM image of fabricated various pore diameters of AAO chip: (a) $15 \mathrm{~nm}$, (b) $35 \mathrm{~nm}$, (c) $55 \mathrm{~nm}$, (d) $75 \mathrm{~nm}$, and (e) $95 \mathrm{~nm}$ pore diameters.

scanning electron microscopy (SEM; Hitachi S-4800) as a structural diagnostic technique. A SEM image of the fabricated AAO chip is shown in Figure 2. The results indicate that the five different pore diameters of AAO $(15,35,55,75$, and $95 \mathrm{~nm}$ ) depended on the type of the anodizing method used. The pore-depth of the fabricated AAO chips was standardized at $1 \mu \mathrm{m}$ in Figure 3. The pore-depth exhibited the best sensitivity at $1 \mu \mathrm{m}$ thickness because the changes in the effective $L$ became larger as the pore-depth decreased [23]. We also found that when the fabrication of the AAO chip's pore-depth was less than $1 \mu \mathrm{m}$ the surface uniformity was compromised.
2.5. Fabrication of the Sensing Membrane. Deposition of gold on the nanostructure has an important role in the induction of LSPR and immobilization of the antibodies on the surface of biocompatible materials. In order to fabricate the sensing membrane, an electron-beam evaporator was used to deposit nickel and gold with thicknesses of $5 \mathrm{~nm}$ and $15 \mathrm{~nm}$, respectively. The evaporator was operated using a pressure setting of $4.0 \times 10^{-6}$ torr and a speed of $0.1 \AA / \mathrm{sec}$. Both gold and silver adhered to nickel and were reported to show improved SPR phenomenon when compared to pure gold and silver [33]. Therefore, by depositing the nickel, we successfully obtained the gold deposited onto AAO membrane. 


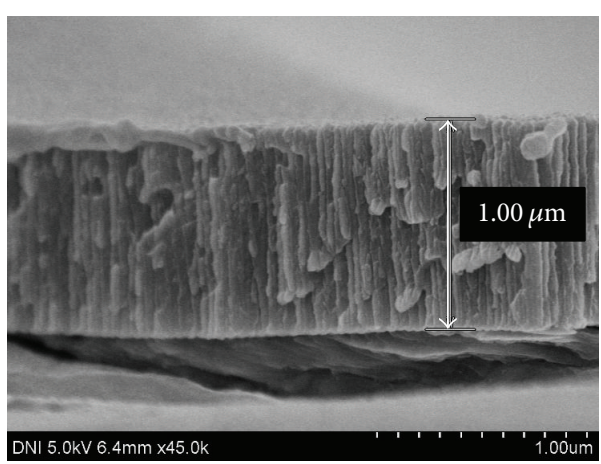

(a)

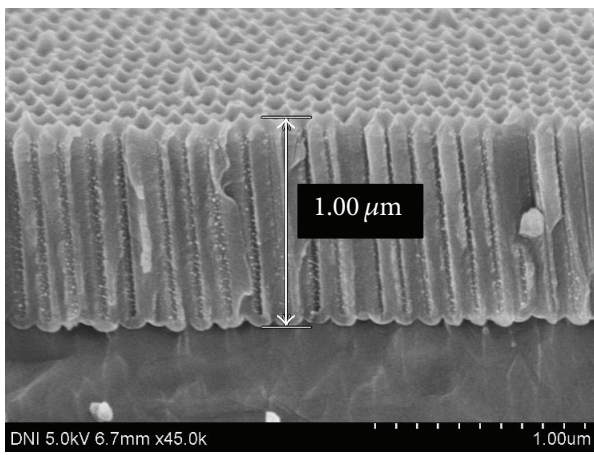

(c)

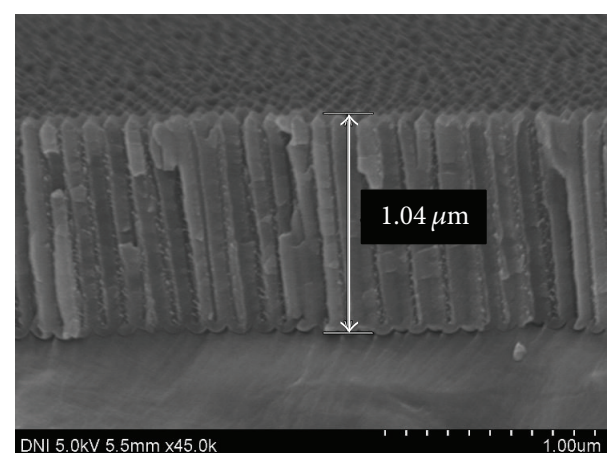

(b)

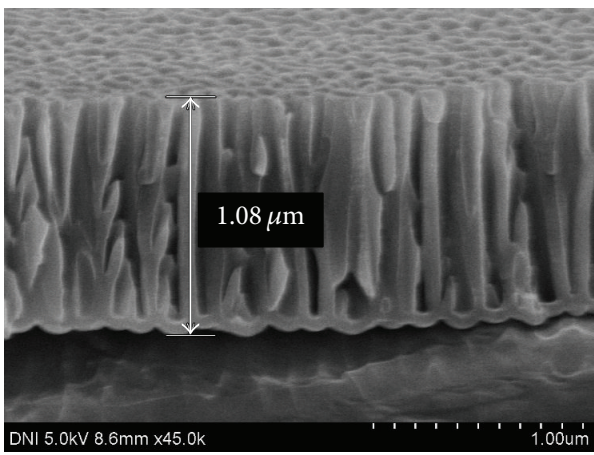

(d)

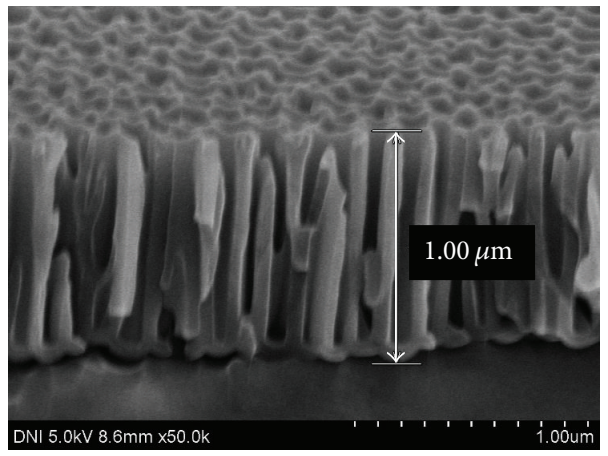

(e)

FIGURE 3: SEM image of a pore-depth of an AAO template with various pore diameters: (a) $15 \mathrm{~nm}$, (b) $35 \mathrm{~nm}$, (c) $55 \mathrm{~nm}$, (d) $75 \mathrm{~nm}$, and (e) $95 \mathrm{~nm}$ pore diameters.

After deposition, alkanethiol SAM was used to immobilize the antibody to the sensing membrane that has strong surface affinity to gold, silver, and copper. One advantage of this procedure is the relatively easy fabrication and the fact that gold, silver, and copper have extremely high affinity to biomaterials. Therefore, the gold deposited AAO chip was immersed in a solution of 11-mercapto-1-undecanol acid in ethanol and incubated for $24 \mathrm{~h} \mathrm{[34].} \mathrm{The} \mathrm{strong} \mathrm{bonding}$ between the gold surface and thiol occurs in the buffer solution, and SAM is completed using a high carboxylic functional group. In order to help bonding of the SAM with carboxyl functional groups and the SAA1 antibody, N-(3dimethylaminopropyl)- $\mathrm{N}^{\prime}$-ethylcarbodiimide hydrochloride (EDC) and N-hydroxysuccinimide (NHS) were prepared in distilled water and dimethyl sulfoxide (DMSO) mixed at identical ratio at concentrations of $200 \mathrm{mM}$ and $50 \mathrm{mM}$, respectively. The mixed solution was stirred for $1 \mathrm{~h}$ until the sensing membrane with SAM was formed. Finally, the SAA1 antibody $(100 \mu \mathrm{g} / \mathrm{mL})$ was stirred for $1 \mathrm{~h}$ to finish the sensing membrane of the AAO chip. The nonspecific binding of antibody was washed out using a buffer solution of Tris- $\mathrm{HCl}$ (pH 7.4). The schematic diagram for SAA1 antibody immobilization process is shown in Figure 4.

2.6. Experimental Setup. The experimental setup used to investigate the detection of lung cancer biomarkers using the nanoporous AAO chip is shown in Figure 5. The measurement system consisted of a spectrometer (QE65000, Ocean Optics Inc.), a white light source (DH-2000-BAL, Ocean Optics Inc.), a reflectance optical probe, an antigen-antibody 


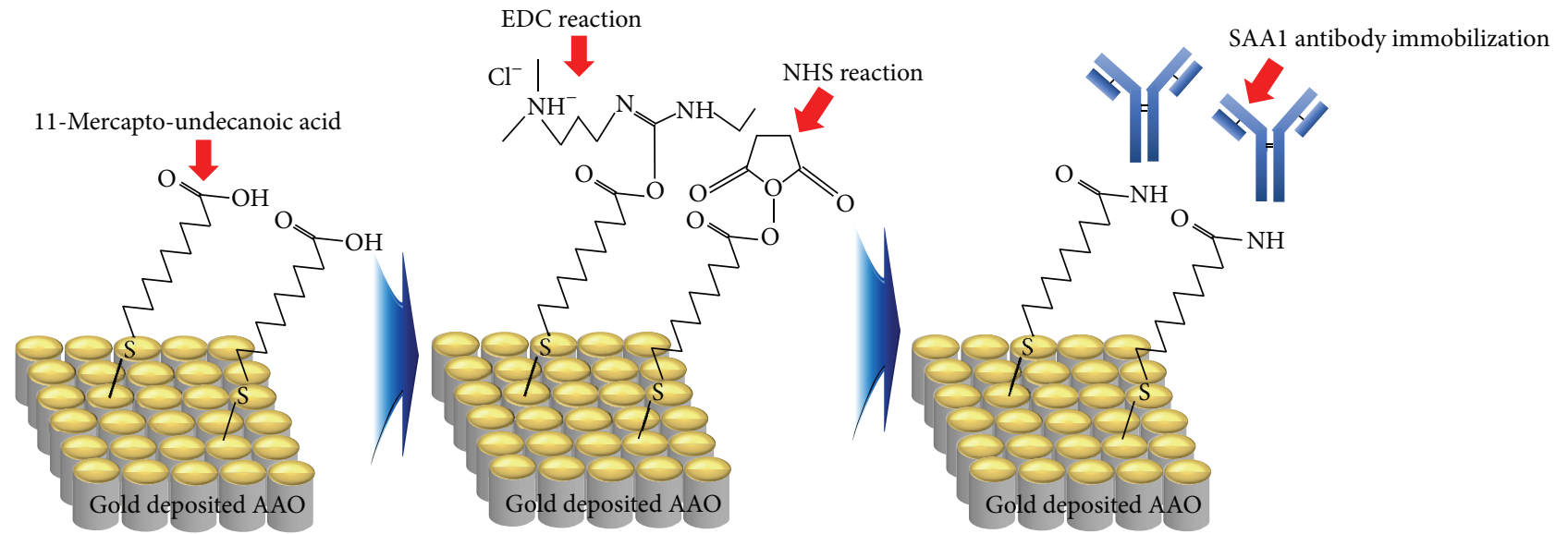

(a)

(b)

(c)

FIGURE 4: Schematic illustration of the surface functionalization steps of gold modified AAO for SAA1 antibody: (a) the formation of selfassembled monolayers (SAMs) of carboxyl-containing thiol followed by (b) covalent attachment of streptavidin of activated SAMs after activation with coupling (NHS/EDC) agents and finally (c) immobilization of SAAl antibody.

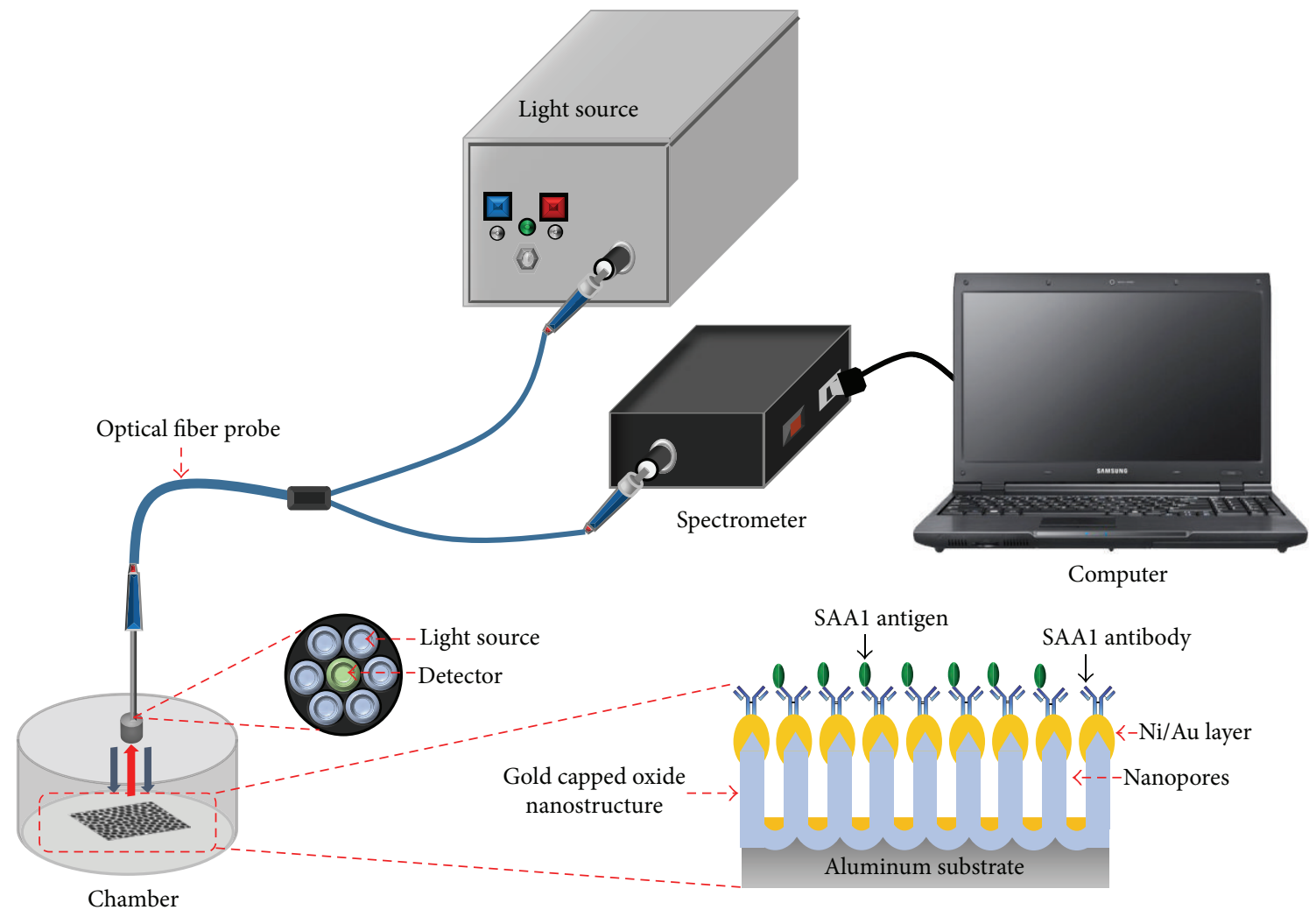

FIGURE 5: Schematic diagram of the lung cancer biomarker measurement system.

reaction chamber, and the AAO chip. The white light source operates in dual mode using deuterium and tungsten-halogen lamps, with the wavelength range of 210 to $1500 \mathrm{~nm}$. The spectrometer has a wide detection range of 200 to $1100 \mathrm{~nm}$ and a maximum resolution of $0.14 \mathrm{~nm}$. The spectrometer is equipped with diverse measurement functions that include transmission, absorption, and reflection. When the light is incident on one direction, the optical prober is connected to the white light source while the other direction is connected to spectrometer. The light is incident in the vertical direction to the sensing membrane through an optical probe fixed to the fabricated chamber. The reflected light is sent to the spectrometer through a light detector using the same optical probe. The reflected light was analyzed using computer spectro-analysis program (Spectrasuit, Ocean Optics Inc.) connected to the spectrometer. 


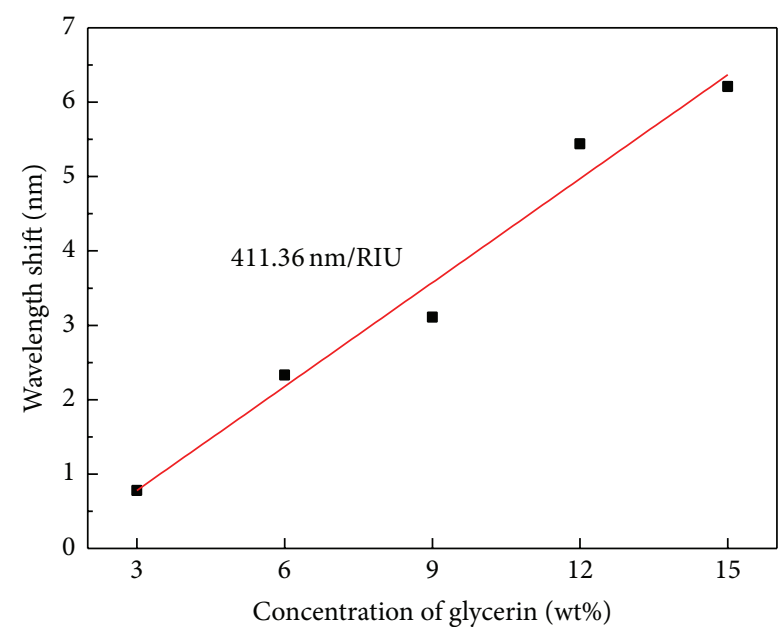

- $0.3 \mathrm{M}$ oxalic acid anodized at $20 \mathrm{~V}$

(a)

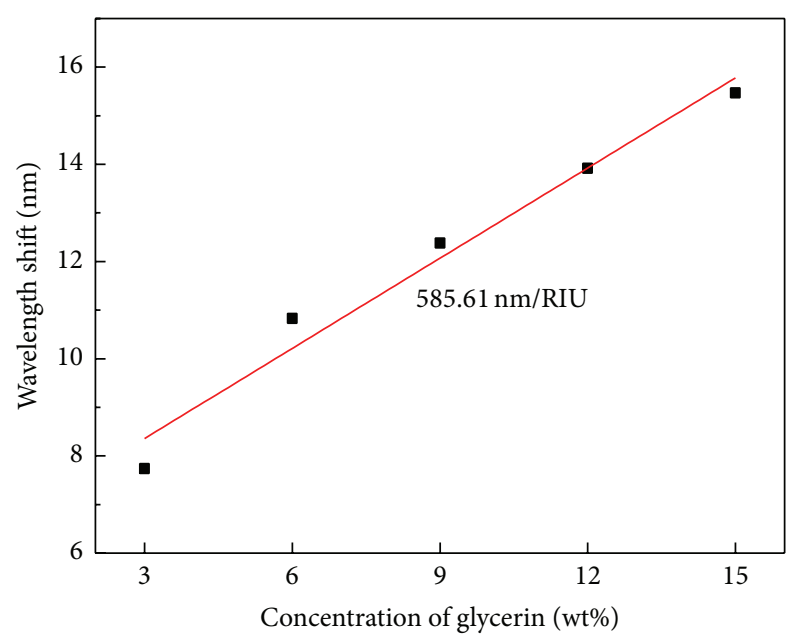

- $0.4 \mathrm{M}$ oxalic acid anodized at $50 \mathrm{~V}$

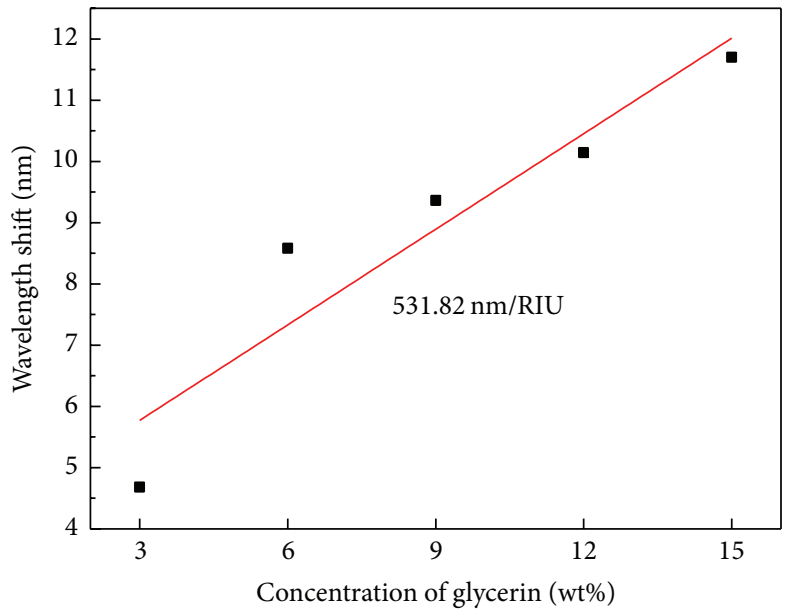

- $0.3 \mathrm{M}$ oxalic acid anodized at $20 \mathrm{~V}$

(b)

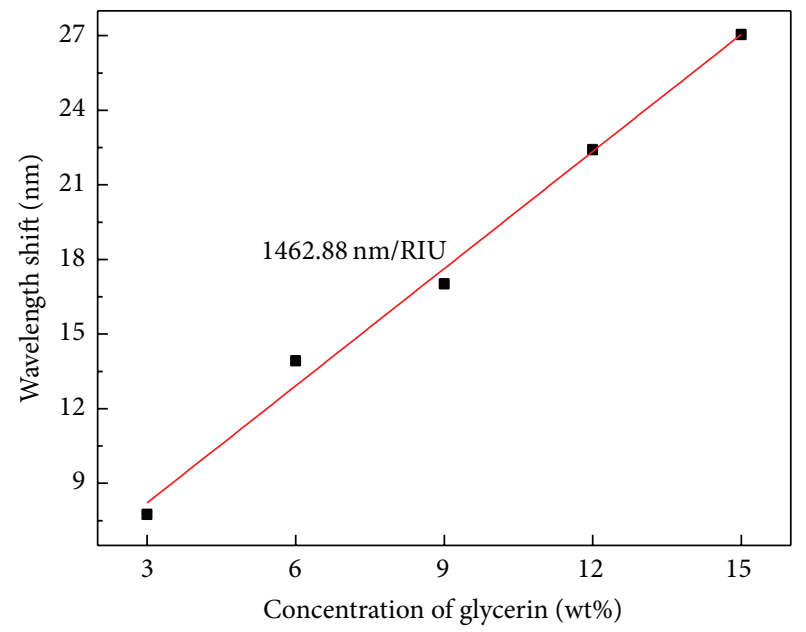

- $0.1 \mathrm{M}$ phosphoric acid anodized at $50 \mathrm{~V}$

(d)

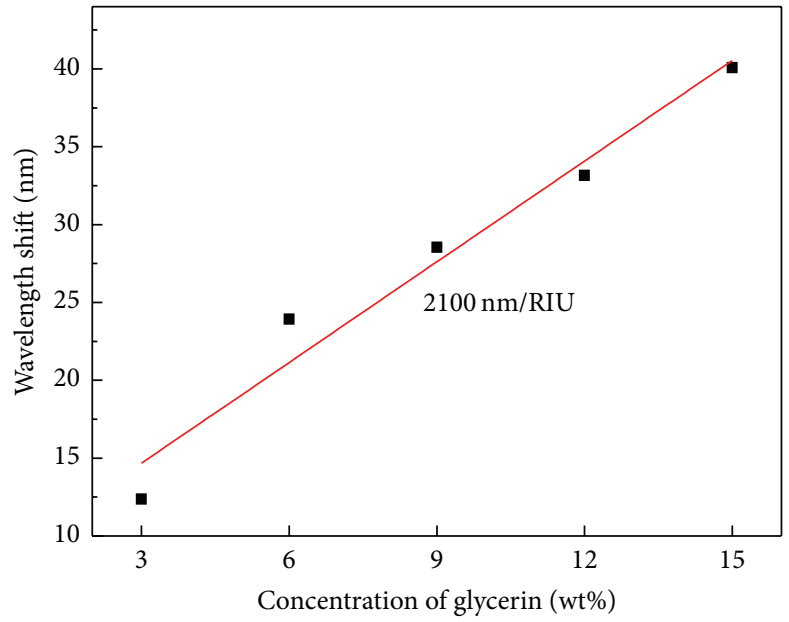

- $1 \mathrm{M}$ phosphoric acid anodized at $50 \mathrm{~V}$

(e)

FIGURE 6: The wavelength shift of various pore diameters of AAO chip according to changes in refractive index of glycerin solutions: (a) $15 \mathrm{~nm}$, (b) $35 \mathrm{~nm}$, (c) $55 \mathrm{~nm}$, (d) $75 \mathrm{~nm}$, and (e) $95 \mathrm{~nm}$. 


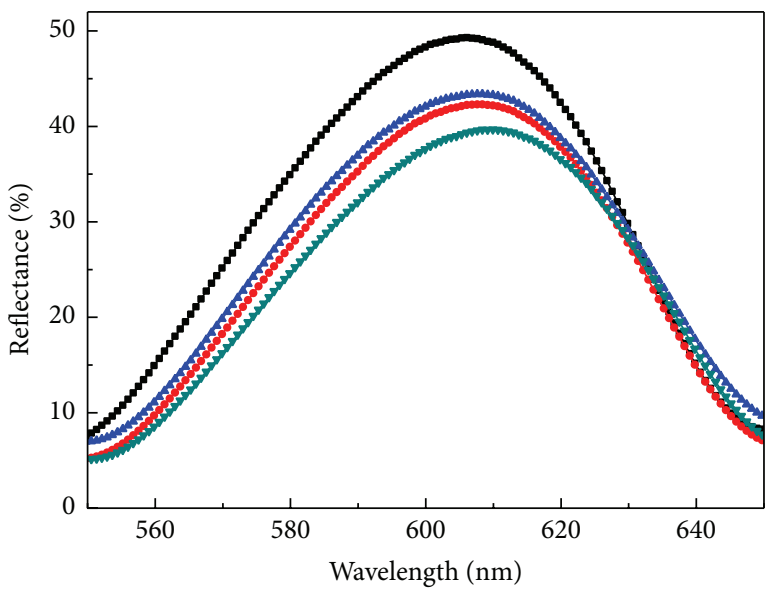

$\begin{array}{ll}\rightarrow \text { SAM } & \rightarrow \text { CRP_antigen }(1 \mu \mathrm{g} / \mathrm{mL}) \\ \rightarrow \text { SAA1_antibody }(100 \mu \mathrm{g} / \mathrm{mL}) & \rightarrow \text { SAA1_antigen }(1 \mu \mathrm{g} / \mathrm{mL})\end{array}$

(a)

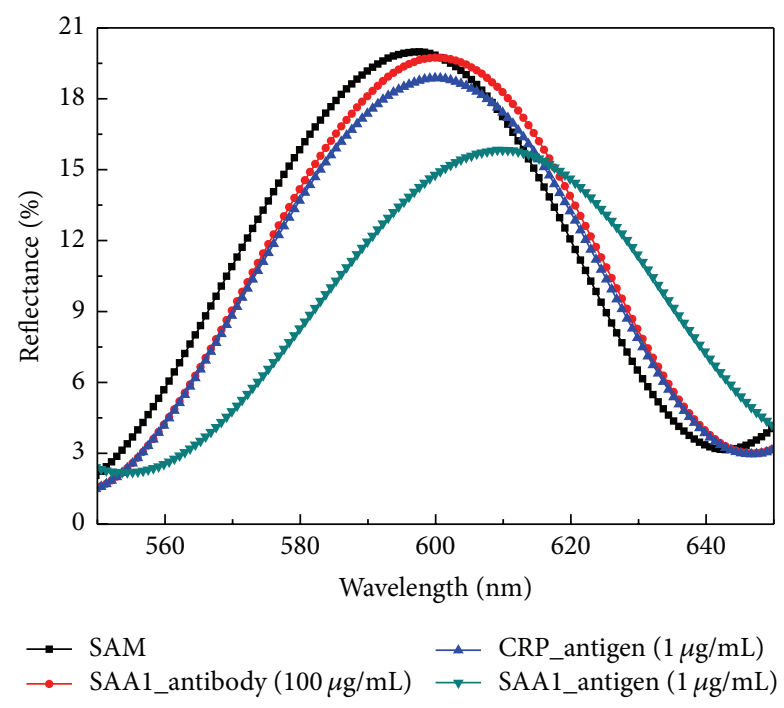

(b)

Figure 7: Selectivity evaluation of the AAO chip: pore diameter of (a) $15 \mathrm{~nm}$ and (b) $95 \mathrm{~nm}$.

\section{Results and Discussions}

3.1. Optical Characteristics of the Sensing Membrane. The optical properties of the sensor chip were verified by detecting changes in the surface refraction. In order to measure optical properties of the change in the interference spectrum against the change in refraction, a glycerin solution was diluted to 3 , $6,9,12$, and $15 \%$ concentrations in distilled water to prepare the refraction solution. Glycerin is commonly used as a RI standard to determine the weight-percent variation. The RI of the refraction solution was performed using an Abbe refractometer (Prisma, CETI, England). The refractive indices were found to be $1.3359,1.3392,1.3430,1.3458$, and 1.3491. After spin-coating, the prepared refraction solution was added to the gold-deposited AAO chip (15, 35, 55, 75, and $95 \mathrm{~nm})$. Next, the optical properties were verified using a measurement system. As a result, the sensitivity was found to be dependent on the increases in the pore diameters of the AAO chip. Figure 6 shows the shifting of the reflective interference signal on the nanoporous AAO chip according to various RIs of the glycerin solutions. The result shows that the sensitivity of the AAO chip with the $95 \mathrm{~nm}$ pore diameter is higher than the sensitivities recorded for other diameter sizes of the pore. The AAO chip with the $95 \mathrm{~nm}$ pore diameter was found to have $2100 \mathrm{~nm} /$ refractive index unit (RIU). We confirmed that the proposed AAO chip can detect RI changes of the surrounding media that result from exposure to the SAA1 antigen.

3.2. Response Characteristics SAA1. To observe the selectivity of the fabricated AAO chip, we evaluated the AAO chips (15 and $95 \mathrm{~nm}$ ) that can distinguish between SAA1 antigen and C-reactive protein (CRP) antigen. The concentration of SAAl antigen used was $1 \mu \mathrm{g} / \mathrm{mL}$, and also the concentration of the CRP antigen was $1 \mu \mathrm{g} / \mathrm{mL}$ in the selectivity experiment. As the results shown in Figure 7, the CRP antigen did not make significant changes in reflectance wavelength, which indicated that no nonspecific binding of the CRP antigen took place on the sensing membrane. However, the SAA1 antigen caused a notable reflectance wavelength shift. The SAA1 antigen wavelengths of the AAO chips with 15 and $95 \mathrm{~nm}$ pore diameters were ranging from $606 \mathrm{~nm}$ to $609 \mathrm{~nm}$ and $597 \mathrm{~nm}$ to $609 \mathrm{~nm}$, respectively. These results revealed the significant response difference between the lineage-specific recognition and the nonspecific reaction. Subsequently, we confirmed other kinds of antigens with the fabricated AAO chip in order to estimate its selectivity.

A nanoporous AAO chip was fabricated to detect immune responses with high sensitivity by combining LSPR with interference. In addition, to evaluate the possibility of its application as a biosensor, the detection properties of the SAA1 antigen were measured. In order to confirm the response characteristics, we applied various concentrations of SAA1 antigen $(10 \mathrm{ag} / \mathrm{mL}, 100 \mathrm{ag} / \mathrm{mL}, 1 \mathrm{fg} / \mathrm{mL}, 1 \mathrm{pg} / \mathrm{mL}$, $1 \mathrm{ng} / \mathrm{mL}$, and $1 \mu \mathrm{g} / \mathrm{mL}$ ) to the sensing membrane that has SAA1 antibodies immobilized on surface of the AAO chip. Nonspecific binding of the detection membrane prepared by immobilizing the SAA1 antibody was removed by washing with Tris- $\mathrm{HCl}$ buffer solution prior to measurement of the reference reflection's wavelength. For improved reliability of the measurement system, the SAA1 antigen was allowed to react with the SAA1 antibody that was immobilized to the sensing membrane by injecting the antigen into the reaction chamber. The shift in the reflection spectrum of the membrane was then measured in real-time. The wavelength shift of reflectance spectra of the nanoporous AAO chip according to concentrations of SAA1 is shown in Figure 8.

Figure 8 shows the reflective interference signal according to various concentrations of SAA1 as a bathochromic shift in the nanoporous AAO chip (15 and $95 \mathrm{~nm}$ ). When comparing the sensitivity of AAO chips of different pore diameters, it was observed that the AAO chip with the $95 \mathrm{~nm}$ pore diameter had larger shifts in the fringe pattern. Moreover, the LOD of 


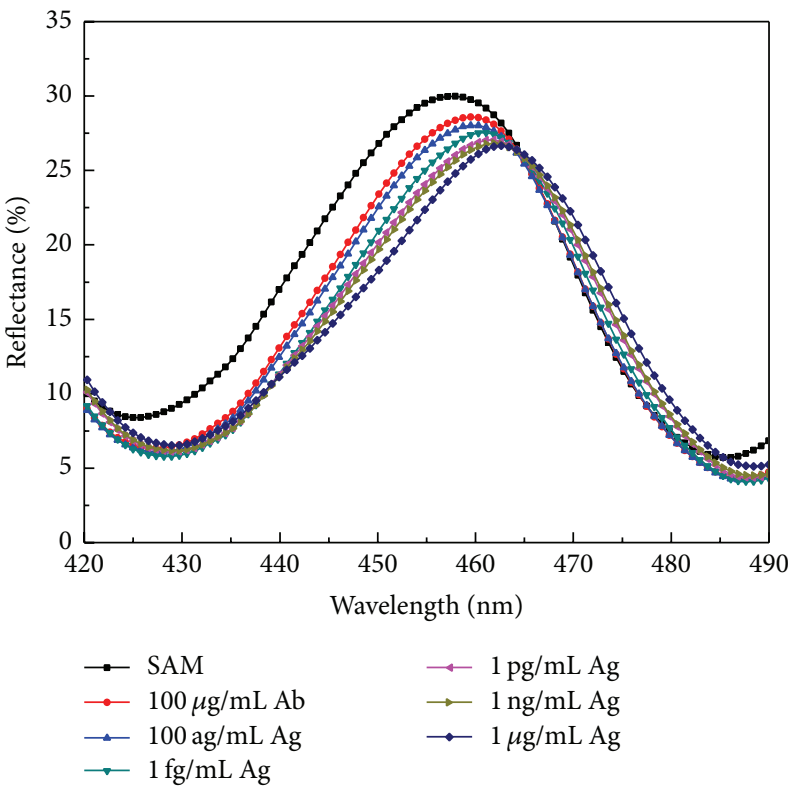

(a)

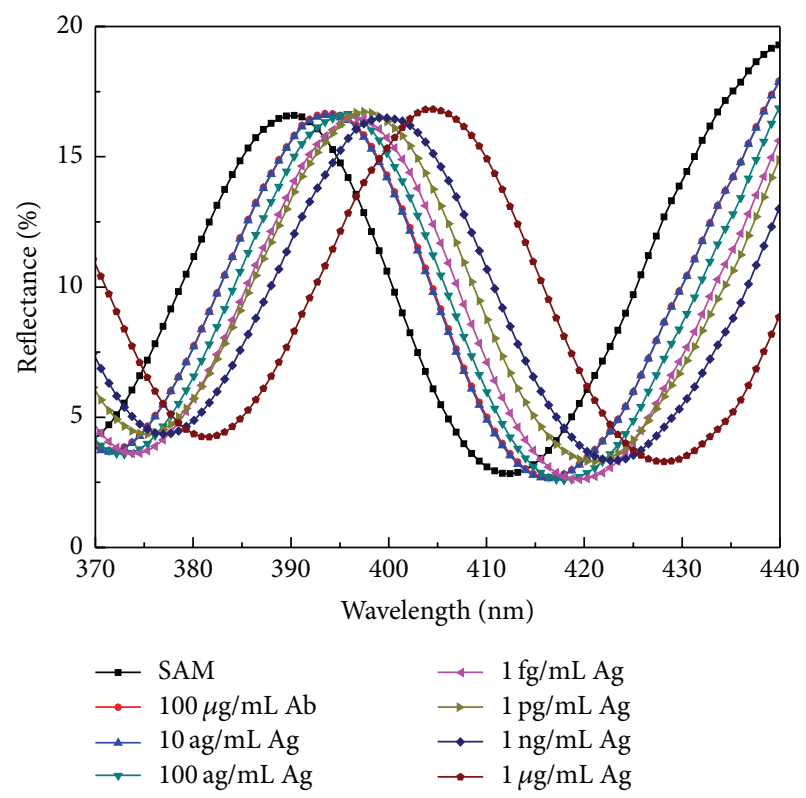

(b)

FIGURE 8: The wavelength shift of reflectance spectra AAO chip according to concentrations of SAAl: pore diameter of (a) $15 \mathrm{~nm}$ and (b) $95 \mathrm{~nm}$.

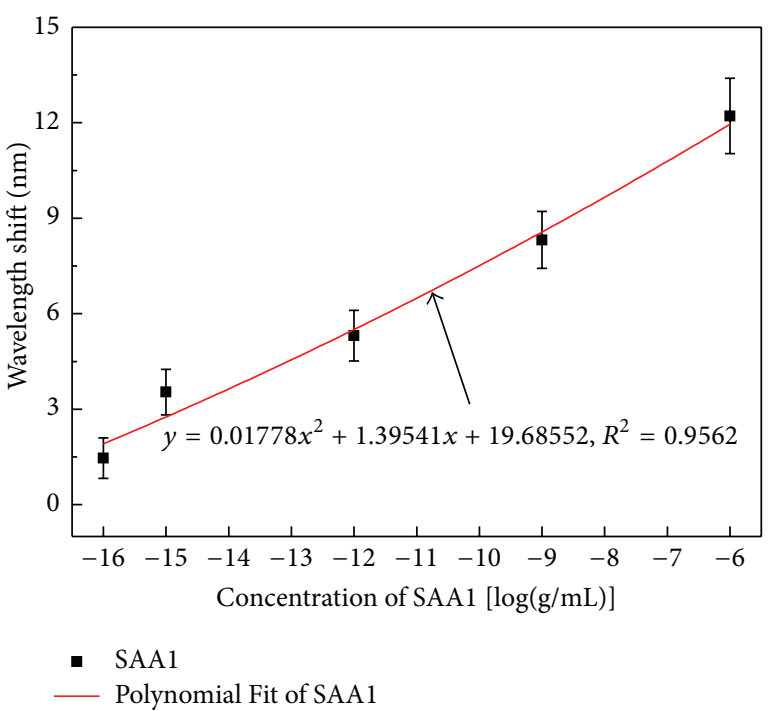

FIGURE 9: The polynomial-regression analysis response according to variations in SAA1 concentrations.

the AAO chips with 15 and $95 \mathrm{~nm}$ pore diameters were found to be $1 \mathrm{fg} / \mathrm{mL}$ and $100 \mathrm{ag} / \mathrm{mL}$, respectively. The experimental measurements were repeated five times. As a result, we confirmed that the nanoporous AAO chip with $95 \mathrm{~nm}$ pore diameter appears to have the highest sensitivity and performance. These experiments were repeated several times. Moreover, the polynomial-regression analysis showed a good correlation coefficient $\left(R^{2}\right), R^{2}=0.9562$. Figure 9 shows the polynomial-regression analysis response to variations in SAA1 concentrations.

\section{Conclusions}

In this study, nanoporous AAO chips, wherein interference and LSPR occur simultaneously, were fabricated, and their response properties for various SAAl concentrations were evaluated in real-time. The AAO chip with excellent surface uniformity was prepared through optimization of the anodization process that could adjust the various pore diameters. The AAO chip quantitatively detected SAA1 with high sensitivity. We obtained high sensitivity $(2100 \mathrm{~nm} / \mathrm{RIU})$ for optical responses to the changes in the surface RI of the nanoporous AAO membrane. In addition, the SAA1 antigen concentration of $10 \mathrm{ag} / \mathrm{mL}$ was also detected via experiments based on immune response. The reflection spectrum properties were analyzed by reacting SAA1 antigen instead of the target biomaterial to evaluate the selectivity of the nanoporous AAO chip and then by analyzing the reflection wavelength. As a result, the sensitivity properties induced by changes in pore diameters were measured to evaluate interference. Best response properties were verified using the device with a $95 \mathrm{~nm}$ pore diameter because of the relative change in effective optical pore diameter of the nanoporous AAO membrane. The fabricated AAO chip was verified so it reacts with high selectivity to the lung cancer-specific biomaterial. The detection limit was found to be $100 \mathrm{ag} / \mathrm{mL}$. In the future, we plan to extend the analysis to include biomarker experiments that use pore diameters larger than $95 \mathrm{~nm}$.

\section{Conflict of Interests}

The authors declare that there is no conflict of interests regarding the publication of this paper. 


\section{Authors' Contribution}

Jae-Sung Lee and Sae-Wan Kim contributed equally to this work.

\section{Acknowledgment}

This work was supported by the National Research Foundation of Korea (NRF) grant funded by the Korea Government (MSIP) (no. NRF2014R1A2A1A11050377).

\section{References}

[1] A. Hasan, M. Nurunnabi, M. Morshed et al., "Recent advances in application of biosensors in tissue engineering," BioMed Research International, vol. 2014, Article ID 307519, 18 pages, 2014.

[2] A. Malima, S. Siavoshi, T. Musacchio et al., "Highly sensitive microscale in vivo sensor enabled by electrophoretic assembly of nanoparticles for multiple biomarker detection," Lab on a Chip, vol. 12, no. 22, pp. 4748-4754, 2012.

[3] M. E. Ali, U. Hashim, S. Mustafa et al., "Nanobiosensor for detection and quantification of DNA sequences in degraded mixed meats," Journal of Nanomaterials, vol. 2011, Article ID 781098, 11 pages, 2011.

[4] A. N. Lad and Y. K. Agrawal, " $\mathrm{SiO}_{2}$-based nanobiosensor monitoring toxicological behavior of Mitoxantrone in vitro," Applied Nanoscience, vol. 4, no. 5, pp. 523-529, 2014.

[5] C. H. Choi, "Integrated nanobiosensor technology for biomedical application," Nanobiosensors in Disease Diagnosis, vol. 2012, article 1, pp. 1-4, 2012.

[6] J. Zhang, C. S. Day, and D. L. Carroll, "Controlled growth of novel hyper-branched nanostructures in nanoporous alumina membrane," Chemical Communications, vol. 7, no. 45, pp. 69376939, 2009.

[7] A. Numnuam, P. Thavarungkul, and P. Kanatharana, "An amperometric uric acid biosensor based on chitosan-carbon nanotubes electrospun nanofiber on silver nanoparticles," Analytical and Bioanalytical Chemistry, vol. 406, no. 15, pp. 37633772, 2014.

[8] R. D. Jean, W. D. Cheng, M. H. Hsiao, F. H. Chou, J. S. Bow, and D. M. Liu, "Highly electrostatically-induced detection selectivity and sensitivity for a colloidal biosensor made of chitosan nanoparticle decorated with a few bare-surfaced gold nanorods," Biosensors and Bioelectronics, vol. 52, no. 15, pp. 111117, 2014.

[9] M. J. Kiani, M. A. A. Razak, F. K. Che Harun, M. T. Ahmadi, and M. Rahmani, "SWCNT-based biosensor modelling for pH detection," Journal of Nanomaterials, vol. 2015, Article ID 721251, 7 pages, 2015.

[10] J. Zhao, X. Zhang, C. R. Yonzon, A. J. Haes, and R. P. Van Duyne, "Localized surface plasmon resonance biosensors," Nanomedicine, vol. 1, no. 2, pp. 219-228, 2006.

[11] I. M. Klotz, Ligand-Receptor Energetics: A Guide for the Perplexed, Wiley, 1997.

[12] D. Kim, K. Kerman, M. Saito et al., "Label-free DNA biosensor based on localized surface plasmon resonance coupled with interferometry," Analytical Chemistry, vol. 79, no. 5, pp. 18551864, 2007.
[13] X. Fan, I. M. White, S. I. Shopova, H. Zhu, J. D. Suter, and Y. Sun, "Sensitive optical biosensors for unlabeled targets: a review," Analytica Chimica Acta, vol. 620, no. 1-2, pp. 8-26, 2008.

[14] M. M. Rahman, A. J. Ahammad, J. Jin, S. J. Ahn, and J. Lee, "A comprehensive review of glucose biosensors based on nanostructured metal-oxides," Sensors, vol. 10, no. 5, pp. 4855-4886, 2010.

[15] B. D. Malhotra and A. Chaubey, "Biosensors for clinical diagnostics industry," Sensors and Actuators B: Chemical, vol. 91, no. 1-3, pp. 117-127, 2003.

[16] M. Saigusa, K. Tsuboi, Y. Konosu, M. Ashizawa, A. Tanioka, and H. Matsumoto, "Highly sensitive local surface plasmon resonance in anisotropic au nanoparticles deposited on nanofibers ," Journal of Nanomaterials, vol. 2015, Article ID 829273, 8 pages, 2015.

[17] P. Schuck, "Reliable determination of binding affinity and kinetics using surface plasmon resonance biosensors," Current Opinion in Biotechnology, vol. 8, no. 4, pp. 498-502, 1997.

[18] D. A. Mann, M. Kanai, D. J. Maly, and L. L. Kiessling, "Probing low affinity and multivalent interactions with surface plasmon resonance: ligands for concanavalin A," Journal of the American Chemical Society, vol. 120, no. 41, pp. 10575-10582, 1998.

[19] J. M. Brockman, A. G. Frutos, and R. M. Corn, "A multistep chemical modification procedure to create DNA arrays on gold surfaces for the study of protein-DNA interactions with surface plasmon resonance imaging," Journal of the American Chemical Society, vol. 121, no. 35, pp. 8044-8051, 1999.

[20] K. C. Popat, G. Mor, C. A. Grimes, and T. A. Desai, "Surface modification of nanoporous alumina surfaces with poly(ethylene glycol)," Langmuir, vol. 20, no. 19, pp. 8035-8041, 2004.

[21] S. Zhao, H. Roberge, A. Yelon, and T. Veres, "New application of AAO template: a mold for nanoring and nanocone arrays," Journal of the American Chemical Society, vol. 128, no. 38, pp. 12352-12353, 2006.

[22] I. Vlassiouk, A. Krasnoslobodtsev, S. Smirnov, and M. Germann, "'Direct' detection and separation of DNA using nanoporous alumina filters," Langmuir, vol. 20, no. 23, pp. 9913-9915, 2004.

[23] S. H. Yeom, O. G. Kim, B. H. Kang et al., "Highly sensitive nanoporous lattice biosensor based on localized surface plasmon resonance and interference," Optics Express, vol. 19, no. 23, pp. 22882-22891, 2011.

[24] S. Yeom, M. Han, B. Kang et al., "Enhancement of the sensitivity of LSPR-based CRP immunosensors by Au nanoparticle antibody conjugation," Sensors and Actuators B: Chemical, vol. 177, pp. 376-383, 2013.

[25] T. Yamada, "Serum amyloid A (SAA): a concise review of biology, assay methods and clinical usefulness," Clinical Chemistry and Laboratory Medicine, vol. 37, no. 4, pp. 381-388, 1999.

[26] M. Kimura, Y. Tomita, T. Imai et al., "Significance of serum amyloid a on the prognosis in patients with renal cell carcinoma," Cancer, vol. 92, no. 8, pp. 2072-2075, 2001.

[27] W. C. Cho, T. T. Yip, W. W. Cheng, and J. S. Au, "Serum amyloid $A$ is elevated in the serum of lung cancer patients with poor prognosis," British Journal of Cancer, vol. 102, no. 12, pp. 1731$1735,2010$.

[28] B. A. Howard, M. Z. Wang, M. J. Campa, C. Corro, M. C. Fitzgerald, and E. F. Patz, "Identification and validation of a potential lung cancer serum biomarker detected by matrixassisted laser desorption/ionization-time of flight spectra analysis," Proteomics, vol. 3, no. 9, pp. 1720-1724, 2003. 
[29] H. Biran, N. Friedman, L. Neumann, M. Pras, and R. ShainkinKestenbaum, "Serum amyloid A (SAA) variations in patients with cancer: correlation with disease activity, stage, primary site, and prognosis," Journal of Clinical Pathology, vol. 39, no. 7, pp. 794-797, 1986.

[30] B. B. Rossi, Optics, Addison-Wesley, 1957.

[31] A. V. Whitney, J. W. Elam, S. Zou et al., "Localized surface plasmon resonance nanosensor: a high-resolution distancedependence study using atomic layer deposition," The Journal of Physical Chemistry B, vol. 109, no. 43, pp. 20522-20528, 2005.

[32] H. Masuda and K. Fukuda, "Ordered metal nanohole arrays made by a two-step replication of honeycomb structures of anodic alumina," Science, vol. 268, no. 5216, pp. 1466-1468, 1995.

[33] H. Liu, B. Wang, E. S. P. Leong et al., "Enhanced surface plasmon resonance on a smooth silver film with a seed growth layer," ACS Nano, vol. 4, no. 6, pp. 3139-3146, 2010.

[34] N. K. Chaki, M. Aslam, J. Sharma, and K. Vijayamohanan, "Applications of self-assembled monolayers in materials chemistry," Journal of Chemical Sciences, vol. 113, no. 5-6, pp. 659-670, 2001. 

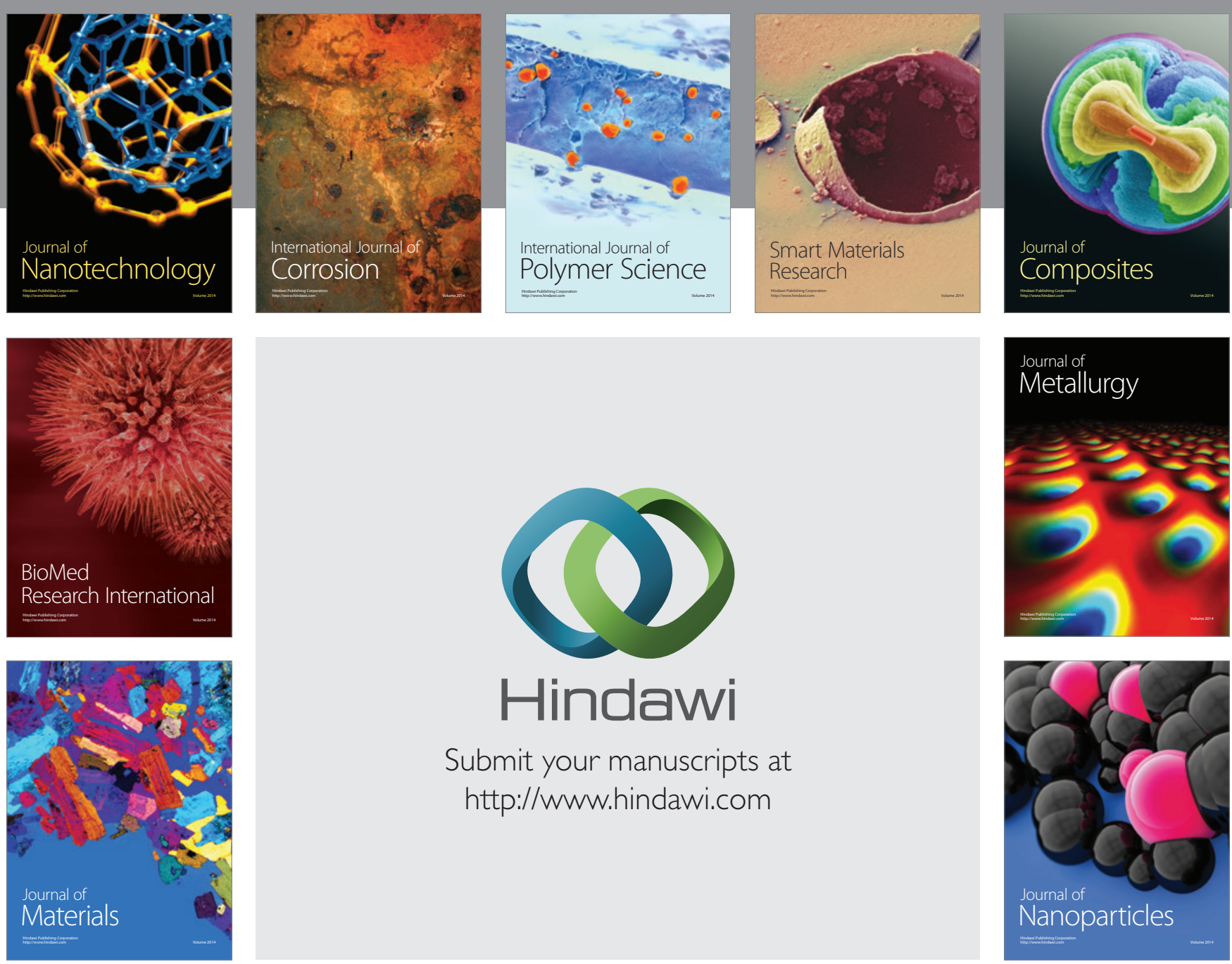

Submit your manuscripts at http://www.hindawi.com
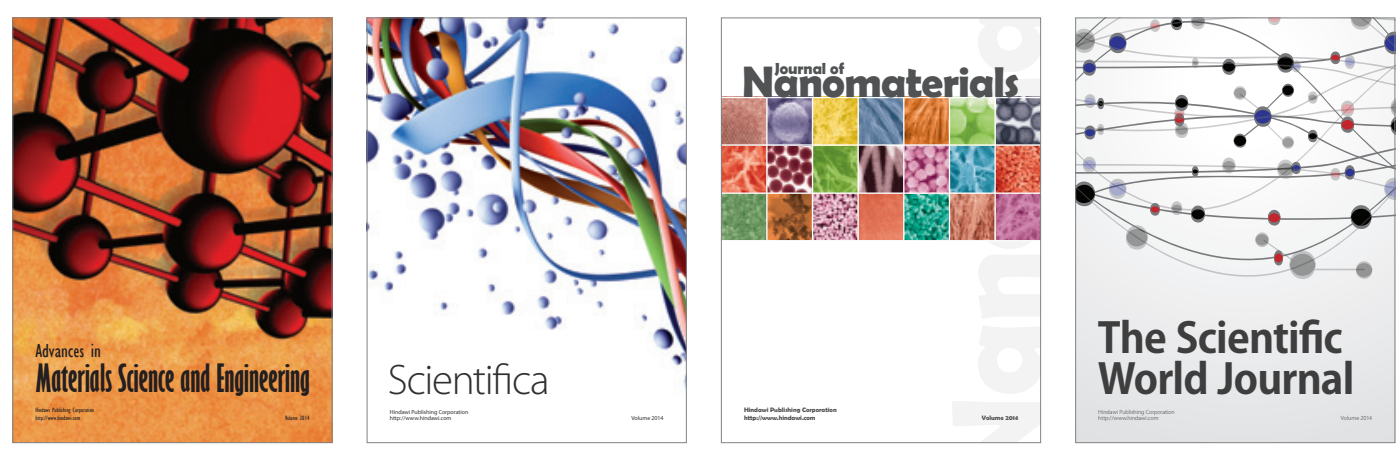

\section{The Scientific World Journal}
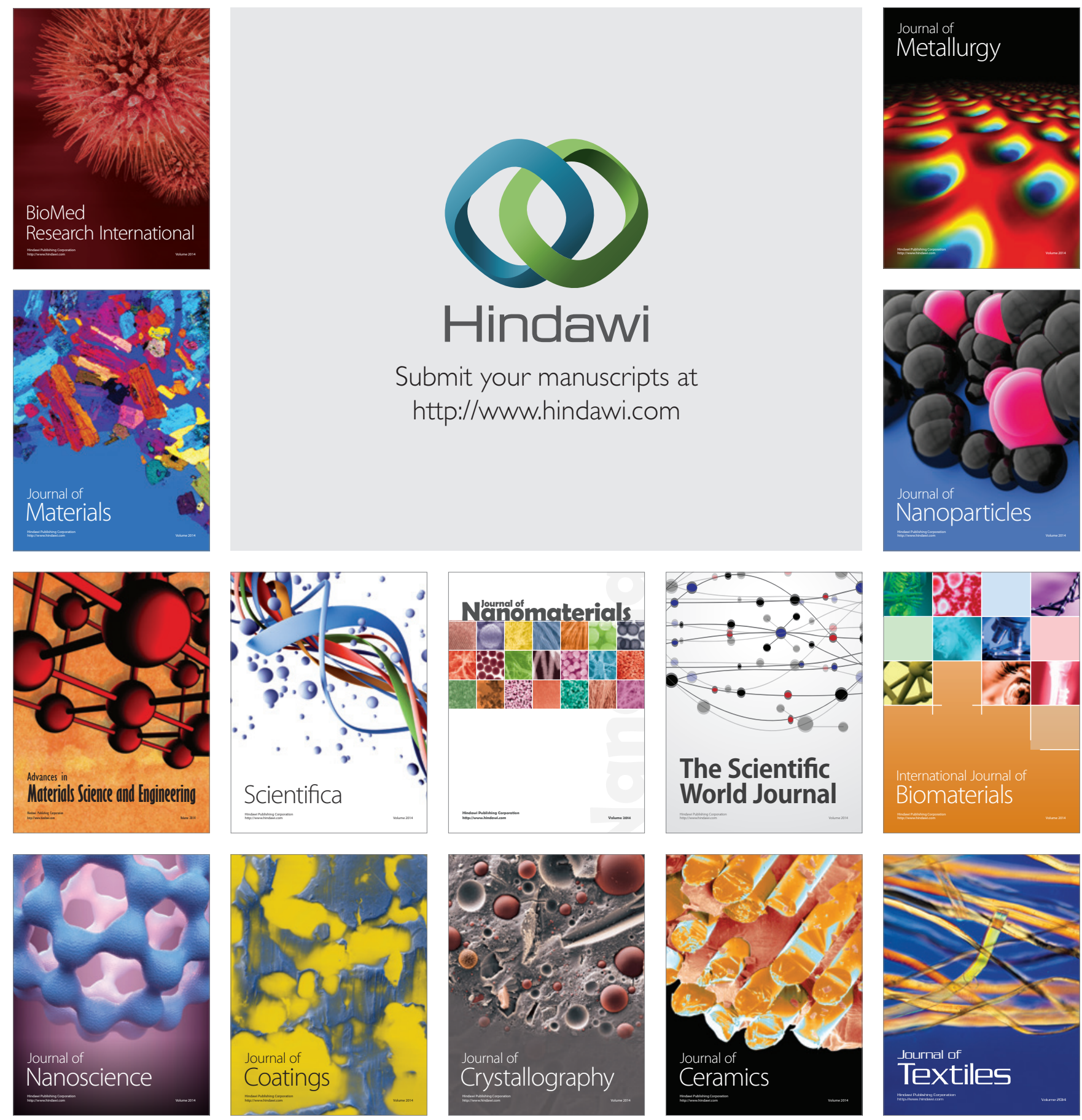